\title{
Systematic Programmed Illumination
}

National Cancer Institute

\section{Source}

National Cancer Institute. Systematic Programmed Illumination. NCI Thesaurus. Code C143954.

A type of light therapy that uses ambient exposure to bluish-white light in a programmed schedule for prevention and treatment of cancer-related fatigue. 